\title{
LEPTOSPIROSE EM CRIANÇAS NO RIO DE JANEIRO
}

\author{
Maria Letícia Santos Cruz, Jarbas Andrade e Martha Maria Pereira
}

\begin{abstract}
A fim de obter dados sobre as manifestações clínicas da leptospirose em crianças, foram revistos 188 exames sorológicos realizados em pacientes com idade entre 0 e 12 anos no Laboratório de Réferência Nacional para Leptospirose (FIOCRUZ-RJ) no período de janeiro de 1983 a junho de 1991. Cinquenta e dois $(27,6 \%)$ soros eram reagentes. Vinte e três $(12,2 \%)$ crianças tinham evidência sorológica de infecção aguda. Os sinais e sintomas mais freqüentemente encontrados nestes casos foram: febre (100\%); mialgias (69,5\%); cefaléia $(52,1 \%)$; ictericia $(47,8 \%)$; vômitos $(34,8 \%)$; dores abdominais, manifestações hemorrágicas e disfunção renal (17,4\%); conjuntivite (13\%); hepatomegalia (4,3\%).
\end{abstract}

Palavras-chaves: Leptospirose. Infância. Formas clínicas. Sorologia. Doença aguda.

A infecção por espiroquetas do gênero Leptospira quando sintomática é acompanhada por manifestaçôes clínicas que surgem de forma isolada ou combinada, com intensidade muito variada. A doença é classicamente descrita como bifásica ${ }^{6} 816$ 171920 . Após início abrupto, geralmente marcado por episódios de febre alta com calafrios e intenso mal estar, a primeira semana cursa com sintomatologia indicativa de quadro infeccioso agudo. Nessa fase os sintomas dominantes são: cefaléia, febre, mialgias, vômitos, diarréia, dor abdominal, rash cutâneo papular ou roseoliforme, congestão conjuntival e tosse $\mathrm{e}^{26}$.

A segunda fase diferencia as formas benignas das graves $^{20}$. Esse período, que costuma ser denominado de tóxico, ictero-tóxico, imune ou de localização ${ }^{12}$, tem duração superior a duas semanas. Vale ressaltar que apenas 5 a $10 \%$ dos pacientes evoluem com icterícia ${ }^{6} 111321$. Nas formas graves, essa é a fase de piora das lesões que se iniciaram no período septicêmico; é quando ocorre a maioria dos óbitos pela infecção ${ }^{20}$. Ashe e cols ${ }^{2}$ consideraram que, para fins práticos, os pacientes que não

\footnotetext{
Serviço de Doenças Infecciosas e Parasitárias do Hospital dos Servidores do Estado e Centro de Referência Nacional para Diagnóstico Laboratorial de Leptospirose do Departamento de Bacteriologia da Fundaçāo Oswaldo Cruz, Rio de Janeiro, RJ. Endereço para correspondência: Dra. Maria Letícia Santos Cruz. Serviço de Doenças Infecciosas e Parasitárias (Anexo 4). Hospital dos Servidores do Estado. R. Sacadura Cabral 179, Saúde, 20221-161 Rio de Janeiro, RJ, Brasil.

Recebido para publicaçāo em 10/12/93.
}

desenvolvem icterícia, a menos que apresentem quadro de meningite, não cursam o segundo período clínico. Os pacientes anictéricos passariam do estágio septicêmico diretamente para a convalescência.

O presente trabalho foi motivado pela marcante escassez de relatos clínicos de crianças com leptospirose, em literatura recente.

\section{MATERIAIS E MÉTODOS}

Visando fornecer material de consulta e discussão a pediatras e infectologistas, realizamos levantamento no arquivo do Centro de Referência Nacional para Diagnóstico de Leptospirose do Departamento de Bacteriologia da Fundação Oswaldo Cruz - RJ. Foram revistos todos os pedidos de exames para crianças de 0 a 12 anos encaminhados a esse laboratório no período de janeiro de 1983 a junho de 1991, inclusive.

Todos os exames solicitados foram acompanhados de uma ficha para identificação e respostas a questões referentes a possíveis fontes de contaminação (fatores de risco), bem como um breve relatório sobre manifestações clínicas.

A técnica utilizada para deteç̧ão de anticorpos contra Leptospira foi a de soroaglutinação microscópica com antígenos vivos, segundo recomendações da Organização Mundial de Saúde 7 . Foram utilizadas 25 cepas de Leptospira, entre os quais sorovares representantes dos sorogrupos mais comuns na área ${ }^{1}$. 
Cruz MLS, Andrade J, Pereira MM. Leptospirose em crianças no Rio de Janeiro. Revista da Sociedade Brasileira de Medicina Tropical 27:5-9, jan-mar, 1994.

\section{RESULTADOS}

No período estudado foram solicitados 188 testes de microaglutinação para Leptospira em soros de crianças de 0 a 12 anos de idade. A maioria das solicitações foi feita nas épocas de epidemia, com destaque para o ano de 1988, quando o Rio de Janeiro sofreu muito devido às fortes chuvas nos meses de verão. Sessenta e quatro por cento dos soros eram de meninos e trinta e seis por cento de meninas.

Cinquenta e dois $(27,6 \%)$ soros foram positivos, dentre estes, $37(71 \%)$ eram de meninos e $15(29 \%)$ de meninas.

Três crianças assintomáticas, cujos exames foram feitos por razões epidemiológicas (por exemplo história de doença na família ou em animal doméstico) apresentavam títulos baixos de anticorpos (Tabela 1).

Dezessete casos sororreagentes e com quadro clínico sugestivo de leptospirose tiveram sua análise prejudicada devido a falta de uma segunda amostra para pareamento. Cinco destes, no entanto, eram muito provavelmente de crianças com infecção aguda, pois na amostra analisada apresentavam anticorpos específicos em títulos de pelo menos 1:3.200 (Tabela 2).

Vinte e três $(12,2 \%)$ crianças sintomáticas mostraram perfil sorológico de doença aguda. Em seis casos, apesar de não ter havido pareamento, o teste revelou soros reagentes para ao menos um sorovar em título igual ou maior que 1:12.800 (Tabela 3).

Estas 23 crianças tinham entre 4 e 12 anos de isiade e ram residentes em bairros da zona norte do município do Rio de Janeiro e da Baixada Fluminense. A Tabela 4 mostra a distribuição por idade e sexo destes casos.

Em 12 das 23 fichas/questionário preenchidas pelos médicos que assistiram a estas crianças, a principal suspeita diagnóstica foi leptospirose $(52,2 \%)$. A Tabela 5 mostra os principais sinais e sintomas encontrács nestes casos.
Tabela 1 - Perfil das crianças assintomáticas com sorologia positiva que tiveram exames solicitados com base em dados epidemiológicos.

\begin{tabular}{|c|c|c|c|}
\hline $\begin{array}{l}\text { Ident } \\
\text { idade } \\
\text { (anos) }\end{array}$ & $\begin{array}{l}\text { cação } \\
\text { sẹxo }\end{array}$ & $\begin{array}{c}\text { Título } \\
\text { predominante }\end{array}$ & Sorovar \\
\hline 3 & $\mathrm{~F}$ & $1: 800$ & patoc \\
\hline 8 & F & $1: 400$ & icterohaemorrhagiae \\
\hline 10 & F & $1: 100$ & patoc \\
\hline
\end{tabular}

Tabela 2 - Perfis sorológicos em apenas uma amostra de sangue de dezessete crianças com suspeita clinica de leptospirose.

\begin{tabular}{|c|c|c|c|}
\hline \multicolumn{2}{|c|}{ Identificação } & \multirow{2}{*}{$\begin{array}{c}\text { Título } \\
\text { predominante }\end{array}$} & \multirow[t]{2}{*}{ Sorovar(es) } \\
\hline $\begin{array}{l}\text { idade } \\
\text { (anos) }\end{array}$ & sexo & & \\
\hline 2 & F & $1: 200$ & 10 \\
\hline 5 & M & $1: 200$ & 1,2 \\
\hline 5 & M & $1: 6400$ & 1 \\
\hline 6 & $M$ & $1: 200$ & 20 \\
\hline 6 & $\mathbf{M}$ & $1: 3200$ & 1,21 \\
\hline 6 & M & $1: 12800$ & 1 \\
\hline 8 & F & $1: 200$ & $1 \mathrm{~A}, 21$ \\
\hline 9 & $\mathrm{M}$ & $1: 800$ & $4,6,17$ \\
\hline 9 & M & $1: 3200$ & 1,8 \\
\hline 10 & M & $1: 1600$ & 6 \\
\hline 10 & $\mathbf{M}$ & $1: 6400$ & 1 \\
\hline 11 & $\mathbf{M}$ & $1: 100$ & $1,1 \mathrm{~A}, 20$ \\
\hline 11 & F & $1: 800$ & 9,21 \\
\hline 11 & $\mathrm{M}$ & $1: 800$ & 11 \\
\hline 12 & $\mathbf{M}$ & $1: 200$ & 21 \\
\hline 12 & $\mathrm{~F}$ & $1: 400$ & 1 \\
\hline 12 & M & $1: 400$ & 1,20 \\
\hline
\end{tabular}

Sorovares: $1=i$ ic ${ }^{\prime}$ ohaemorrahagiae (cepa 3294); $1 \mathrm{~A}=$ icterohaemorhagiae (cepa RGA); 2 = copenhageni; $4=$ canicola $; 6=$ pyrogenes $; 8=$ autumnalis; $9=$ sentot; $10=$ djasiman $; 11=$ australis $; 17=$ saxkoebing ; $20=$ panama $; 21=$ patoc . 
Cruz MLS, Andrade J, Pereira MM. Leptospirose em crianças no Rio de Janeiro. Revista da Sociedade Brasileira de Medicina Tropical 27:5-9, jan-mar, 1994.

Tabela 3 - Perfis sorológicos de vinte e três casos de doença aguda diagnosticados sorologicamente.

\begin{tabular}{|c|c|c|c|}
\hline \multicolumn{2}{|c|}{ Identificação } & \multicolumn{2}{|c|}{ Amostra } \\
\hline $\begin{array}{l}\text { idade } \\
\text { (anos) }\end{array}$ & sexo & $1^{\mathrm{a}}$ & $2^{a}$ \\
\hline 4 & $\mathbf{M}$ & $1: 3200$ ictero & 1:3200 ictero \\
\hline 6 & M & $1: 800$ ictero & $1: 25600$ ictero \\
\hline 6 & $M$ & 1:12800 ictero & - \\
\hline 7 & M & 1:200 ictero & $1: 6400$ ictero \\
\hline 8 & $\mathbf{M}$ & 1:12800 ictero & $1: 6400$ ictero \\
\hline 8 & M & $1: 12800$ ictero & $1: 3200$ ictero \\
\hline 9 & $\mathrm{M}$ & 1:25600 ictero & - \\
\hline 9 & $\mathbf{M}$ & 1:6400 ictero & $1: 6400$ ictero \\
\hline 9 & $\mathbf{M}$ & 1:6400 ictero & 1:6400 ictero \\
\hline 10 & $\mathbf{M}$ & 1:800 ictero & $1: 12800$ ictero \\
\hline 10 & $\mathbf{M}$ & 1:12800 ictero & $1: 102400$ ictero \\
\hline 10 & F & $1: 12800$ ictero & $1: 25600$ ictero \\
\hline 11 & $\mathbf{M}$ & $<1: 100$ ictero & $1: 600$ patoc \\
\hline$i 1$ & 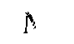 & $1: 12800$ ictero & - \\
\hline 11 & N & 1:51200 ictero & $1: 51200$ ictero \\
\hline 11 & $M$ & $1: 51200$ ictero & - \\
\hline $1 ?$ & $\mathrm{M}$ & $<1: 100$ canicola & 1:3200 patoc \\
\hline 12 & $\mathbf{M}$ & $<1: 100$ ictero & 1:6400 ictero \\
\hline 12 & $\mathrm{~F}$ & 1:12800 ictero & $1: 25600$ ictero \\
\hline 12 & $\mathbf{M}$ & $1: 12800$ ictero & $1: 12800$ ictero \\
\hline 12 & $\mathrm{M}$ & $1: 25600$ ictero & - \\
\hline 12 & $M$ & 1:25600 ictero & - \\
\hline 12 & F & $1: 25600$ ictero & - \\
\hline
\end{tabular}

Tabela 4 - Distribuição por idade e sexo dos vinte e três casos de leptospirose em crianças diagnosticadas por sorologia no Laboratório Leptospirose FIOCRUZde janeiro/83 a junho/ 91.

\begin{tabular}{lll}
\hline $\begin{array}{l}\text { Idade } \\
\text { (anos) }\end{array}$ & Meninos & Meninas \\
\hline
\end{tabular}

\begin{tabular}{rlll}
\hline 4 & 1 & 0 & 1 \\
5 & 0 & 0 & 0 \\
6 & 2 & 0 & 2 \\
7 & 1 & 0 & 1 \\
8 & 2 & 0 & 2 \\
9 & 3 & 0 & 3 \\
10 & 2 & 1 & 3 \\
11 & 4 & 0 & 4 \\
12 & 5 & 2 & 7 \\
\hline Total & 20 & 3 & 23 \\
\hline
\end{tabular}

Tabela 5 - Principais sinais e sintomas relatados por médicos assistentes nos questionários das vinte e três crianças com evidência sorológica de doença aguda.

\begin{tabular}{lc}
\hline Sinal/Sintoma & $\begin{array}{c}\text { Proporção encontrada } \\
(\%)\end{array}$ \\
\hline Febre & 100,0 \\
Mialgia & 69,5 \\
Cefaléia & 52,1 \\
Ictericia & 47,8 \\
Vômitos & 34,8 \\
Dor abdominal & 17,4 \\
Manifestação hemorrágica & 17,4 \\
Insuficiência renal & 17,4 \\
Congestão conjuntival & 13,0 \\
Hepatomegalia & 4,3 \\
\hline
\end{tabular}

\section{DISCUSSÃO}

\section{O diagnóstico de leptospirose por sorologia}

$\mathrm{Na}$ Tabela 2 vemos a relação de casos cujo diagnóstico sorológico não pode ser feito devido à falta de segunda amostra de sangue para pareamento. Este cuidado é particularmente importante em áreas onde a infecção é endêmica, como no Rio de Janeiro. Nesta situação é comum encontrarmos títulos aparentemente expressivos na população geral (assintomáticos).

É possível que as crianças que exibiam reações de microaglutinação positivas em diluições iguais ou maiores que 1:3200 para pelo menos um sorovar, fossem portadoras de doença aguda. Quanto aos que tiveram teste positivo até 1:1600, tudo o que podemos dizer é que houve infeç̧ão mas não devemos aceitar esta reatividade como prova diagnóstica de doença atual. Por outro lado não devemos esquecer a possibilidade da bateria de antígenos utilizada não conter representante homólogo ao sorovar infectante. $\mathrm{Na}$ interpretação desses dados temos ainda a considerar os possíveis efeitos das medidas terapêuticas (como por exemplo o uso de antibióticos) sobre os anticorpos circulantes.

\section{Quadro clínico da leptospirose na infância}

Levantamentos sobre manifestações clínicas no curso da leptospirose mostram que a grande maioria dos pacientes ${ }^{9}$ apresenta sintomas inespecíficos, de 
Cruz MLS, Andrade J, Pereira MM. Leptospirose em crianças no Rio de Janeiro. Revista da Sociedade Brasileira de Medicina Tropical 27:5-9, jan-mar, 1994.

intensidade e duração variáveis ${ }^{4} 91518$. Os levantamentos gerais mostram como sintomas mais comuns, febre, cefaléia, mialgias, alterações gastrointestinais, hiperemia conjuntival e sintomas respiratórios $^{6} 1518$.

No que se refere à apresentação clínica dos casos em crianças, os poucos trabalhos publicados mostram que, de forma geral, os sinais e sintomas são os mesmos dos adultos ${ }^{5}{ }^{14}$. A freqüência dos achados, no entanto, parece diferente das séries compostas por adultos. Galvão e cols ${ }^{10}$, analisaram 37 casos de leptospirose em pacientes com menos de 15 anos de idade, encontrando como principais achados: náuseas e/ou vômitos $(94,5 \%)$; febre $(86,4 \%)$; icterícia $(51,3 \%)$; mialgias $(48,8 \%)$; cefaléia e astenia (45,9\%); anorexia $(32,4 \%)$; diarréia $(29,7 \%)$; dor abdominal e sonolência $(24,3 \%)$; tosse $(13,5 \%)$. Os sintomas hemorrágicos nessa população estiveram presentes apenas em $9 \%$ dos pacientes.

Wong e cols ${ }^{22}$, descreveram 9 casos em crianças de 2 a 14 anos de idade. Todos os pacientes tinham febre alta e anorexia. Os outros sintomas mais observados foram: dor abdominal, icterícia, conjuntivite, linfoadenopatia cervical; cada um tendo acometido 7 dos enfermos. Náuseas e/ou vômitos foram registrados em 6 casos, assim como o rash cutâneo. Este trabalho é muito enriquecedor porque chama atenção para apresentações clínicas que não são relatadas em adultos com leptospirose. Cinco pacientes apresentavam hidropsia de vesícula biliar, sem obstrução de ductos biliares (colecistite acalculosa). Em uma das crianças, o rash cutâneo, inicialmente maculopapular, evoluiu para lesões purpúricas. As lesões foram biopsiadas e revelaramse resultantes de vasculite acometendo circulação arterial e venosa.

As vinte e três crianças que tiveram diagnóstico de doença aguda entre 1983 e 1991 , cadastradas no Laboratório de Leptospirose da FIOCRUZ, de forma geral, apresentaram sintomas em freqüência semelhante à encontrada na literatura.

Nesta série a icterícia foi relatada em $47,8 \%$ dos casos. Galvão e cols ${ }^{10}$, em 1968, encontraram icterícia em 51,3\% das crianças hospitalizadas com leptospirose. A alta incidência de icterícia nas duas séries de crianças deve refletir tendenciosidade nas amostras, que são compostas principalmente por crianças hospitalizadas em processo de esclarecimento diagnóstico. Achamos possível que o diagnóstico de leptospirose seja mais freqüentemente aventado quando há constatação de icterícia.

Dados nacionais atualizados sobre casos de leptospirose anictérica são ainda mais escassos. Esta forma de apresentação é muito parecida com o dengue. Em 1990, durante inquérito sorológico que realizamos em escolares de São João de Meriti (Rio de Janeiro) ${ }^{5}$, observamos 30 crianças soropositivas com história passada de quadro clínico sugestivo de leptospirose anictérica. Essas crianças, na época, foram levadas para atendimento médico e em todos os casos o diagnóstico foi de dengue. Ao testarmos esses sangues, apenas 5 apresentaram anticorpos para vírus do dengue (tipos 1 e 2). Este achado nos faz pensar na necessidade e importância de um maior aprofundamento no estudo sobre formas clínicas de leptospirose na infância. Um trabalho que vise a esclarecer essa questão, pressupõe vigilância e busca, objetivando o diagnóstico de casos febris não só em pacientes internados, mas, principalmente durante atendimento ambulatorial. Um estudo laboratorial, com tentativa de isolamento do germe durante os primeiros dias de doença e pesquisa seriada de anticorpos, é fundamental. Só como auxílio dessas observações, se poderá descrever melhor o espectro clínico e epidemiológico da leptospirose em nosso meio.

\section{SUMMARY}

In order to obtain data about clinical manifestations of symptomatic leptospiral infection in children, the authors reviewed 188 microscopic agglutination tests performed on sera of patients aged 0 to 12 years, made at the National Reference Laboratory of Leptospirosis (F1OCRUZ-RJ) from January 1983 to June 1991. Fifty two (27.6\%) sera were positive. Twenty three (12.2\%) children had serological evidence of acute infection. The most frequent signs and symptoms of these 23 cases were: fever (100\%); myalgia (69.5\%); headache (52.1\%); jaundice $(47.8 \%)$; vomit $(34.8 \%)$; abdominal pain, hemorrhagic manifestations and impaired renal function (17.4\%); conjunctivitis (13\%); hepatomegaly (4.3\%).

Key-words: Leptospirosis. Childhood. Clinical manifestation. Serology. Acute disease. 
Cruz MLS, Andrade J, Pereira MM. Leptospirose em crianças no Rio de Janeiro. Revista da Sociedade Brasileira de Medicina Tropical 27:5-9, jan-mar, 1994.

\section{REFERÊNCIAS BIBLIOGRÁFICAS}

1. Andrade J, Brandão AP. Contribuição ao conhecimento da epidemiologia da leptospirose humana, com especial referência ao Grande Rio, Brasil, no período de 1970 a 1982 . Memórias do Instituto Oswaldo Cruz 82:91-100, 1987.

2. Ashe WMF, Pratt-Thomas HR, Kumpe CW. Weil's disease: a complete review of american literature and an abstract of the world literature. Seven case reports. Medicine 20:145-210, 1941.

3. Barbosa LT. Formes cliniques de l'infection á "Spirochaeta icterohemorrhagiae" chez l'enfant. Medicina-Cirurgia-Pharmacia 37:163-222, 1939.

4. Berman SJ, Tsai C, Holmes K, Fresh JW, Watten RH. Sporadic anicteric leptospirosis in South Vietnam. Annals of Internal Medicine 79:167-173, 1973.

5. Cruz MLS. Leptospirose na infância: inquérito para prevalência e fatores de risco em escolares de São João de Meriti, Rio de Janeiro. Tese de Mestrado, Fundação Oswaldo Cruz, 1992.

6. Edwards GA, Down BM. Human leptospirosis. Medicine 39:117-152, 1960.

7. Faine S, Adler B, Palit A. Clinical, serological and biological properties of a serotype-specific polysaccharide antigen in Leptospira. Australian Journal of Experimental Biology and Medical Science 52:311-319, 1974.

8. Farrar WE. Leptospira species (Leptospirosis). In: Mandell GL, Douglas RG, Bennett JE (eds) Principles and Practice of Infectious Diseases. Churchil Livingstone p.1813-1816, 1990.

9. Feigin RD, Anderson DC. Leptospirosis. In: Feigin RC, Cherry JD (eds) Textbook of Pediatric Infectious Diseases. WB Saunders Company p.895-911,1981.

10. Galvão PAA, Scheinberg MA, Pereira Jr W, Fucs M, Sonnewend JPAS, Farhat CK, Correa MOA. Leptospirose na infância. Pediatria Prática 39:155$160,1968$.

11. Health $\mathrm{Jr} \mathrm{CW}$, Alexander AD, Galton MM. Leptospirosis in the United States. Analysis of 483 cases in man, 1949-1961. New England Journal of
Medicine 273:857-922, 1965.

12. Jeghers HJ, Houghton JD, Foley JA. Weil's Disease: report of a case and review of recent literature. Apud: Ashe WF, Pratt-Thomas HR, Kumpe CW, 1941. Weil's Disease: a complete review of american literature and an abstract of the world literature. Seven ease reports. Medicine 20:145-210, 1935.

13. Lecour $H$, Miranda $M$, Magro $C$, Rocha $A$, Gonçalves V. Human leptospirosis - a review of 50 cases. Infection 7:8-12, 1989.

14. Lombardi F. Considerações sobre alguns casos de icterícia leptospirilar na infância. Revista Brasileira de Medicina 2:633-636, 1945.

15. McCrumb FR, Stockard JL, Robinson CR, Turner LH, Levis DG, Maisey CW, Kelleher MF, Gleiser CA, Smadel JE. Leptospirosis in Malaya. The American Journal of Tropical Medicine and Hygiene 6:238-256, 1952.

16. Noleto PA. Hepatite por leptospira. Tese de Professor Titular, Universidade do Estado do Rio de Janeiro, Rio de Janeiro, 1979.

17. Prata A, Teixeira R. Leptospirose na Bahia. Hospital 53:827-837, 1958.

18. Russel RWR. Clinical features of Tropical Leptospirosis. Annals of Tropical Medicine and Parasitology 53:416-420, 1959.

19. Sanford JP. Leptospirosis. In: Brownwald E, Isselbacher KJ, Peterdorf RG, Wilson JD, Martin JB, Fauci AS. Harrison's Principles of Internal Medicine 11Ed. McGraw-Hill Book Co, New York, 1987.

20. Silva JJP. Formas graves de leptospirose contribuição ao seu estudo clínico. Tese de Mestrado, Universidade Federal do Rio de Janeiro, Rio de Janeiro, 1974.

21. Turner LH. Leptospirosis. Transactions of the Royal Society of Tropical Medicine and Hygiene 61:842$855,1967$.

22. Wong ML, Kaplan S, Dunkle LM, Stevchenber BW, Feigin RD. Leptospirosis: A childhood disease. Journal of Pediatrics 90:532-537, 1977. 